\title{
Rolling friction of a hard cylinder on a viscous plane
}

\author{
Thorsten Pöschel ${ }^{1}$, Thomas Schwager ${ }^{1}$, and Nikolai V. Brilliantov ${ }^{1,2}$ \\ ${ }^{1}$ Humboldt-Universität zu Berlin, Institut für Physik, Invalidenstraße 110, \\ D-10115 Berlin, Germany \\ ${ }^{2}$ Moscow State University, Physics Department, Moscow 119899, Russia
}

(August 10, 2021)

\begin{abstract}
The resistance against rolling of a rigid cylinder on a flat viscous surface is investigated. We found that the rolling-friction coefficient reveals strongly non-linear dependence on the cylinder's velocity. For low velocity the rolling-friction coefficient rises with velocity due to increasing deformation rate of the surface. For larger velocity, however, it decreases with velocity according to decreasing contact area and deformation of the surface.

PACS numbers: 46.30.Pa, 62.40.+i, 81.40.Pq
\end{abstract}

\section{INTRODUCTION}

The effect of rolling friction has been investigated by many scientists according to its great importance in engineering (e.g. [1]) and physical science (e.g. [2]). Scientific publications on rolling friction range back to, at least, 1785 when Vince described systematic experiments to determine the nature of friction laws [3].

It is known that surface effects such as adhesion (e.g. [4]), electrostatic interaction (e.g. [5]), and other surface properties (e.g. [6]) may have strong influence on rolling friction. For viscoelastic materials, however, it was argued that rolling friction is due very little to surface interactions, i.e. the major part is due to deformation losses within the bulk of the material [7,8]. Under this assumption Greenwood et al. [7] calculated the rolling friction coefficient for a hard sphere rolling on a soft plane. The deformation in the bulk was assumed to be completely plastic. Then an empirical coefficient was introduced to account for the incomplete recover of the material. Recently a similar problem has been addressed in [9] where the rolling friction coefficient for a soft sphere on a hard plane has been derived as a firstprinciple continuum-mechanics expression. This coefficient has been found within a quasi-static approach 10 as a function of the viscous and elastic constants of the sphere material without introducing phenomenological parameters.

In the case of a soft sphere rolling on a hard plane [9] the contact surface between the bodies is flat. This allows for the application of Hertz's contact theory. In the opposite case of a hard sphere or cylinder on a viscous plane which we address here, this assumption is not justified since the plane deforms in such a way that its shape follows the shape of the rolling body in the area of contact. This complicated shape of the contact surface excludes the direct application of Hertz's contact theory and may violate Hertz's contact law which relates the force acting between the interacting bodies to their deformation.

The velocity dependence of the rolling friction coefficient originates from the fact that the deformation of the surface varies with the velocity of the rolling body. For small velocities the viscous stress, proportional to the deformation rate, is small. In this case the deformation of the plane (measured by a depth $h$ of which the body penetrates the surface, see Fig. 11) is determined mainly by the elasticity of the plane and by the weight of the body. On the other hand at very large velocities the viscous stress becomes comparable to the elastic stress. As a result the plane supports the rolling body at significantly smaller deformations. This leads to decreasing penetration depth $h$, hence, less energy may be required to deform the surface. In this case one observes decreasing resistance to rolling with increasing velocity. Mainly because of the complicatedly shaped contact area it is not possible to treat these effects within the first-principle continuummechanics description, hence a simpler model will be considered. As shown in the following this model reflects the most important properties of the problem addressed.

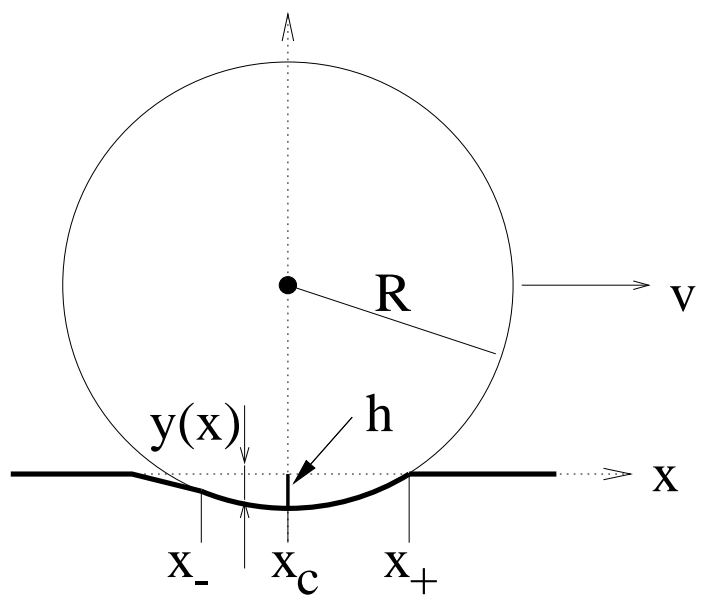

FIG. 1. Sketch of the system: A rigid cylinder rolls on a plane built up of independently moving damped springs. According to the motion of the cylinder the surface is deformed and mechanical energy is lost due to its damped motion. This dissipation of energy can be understood as rolling friction. 


\section{THE MODEL}

We investigate the resistance against rolling of a hard cylinder on a soft plane. Consider a cylinder of radius $R$, length $L \gg R$ and mass per unit length $M$ which rolls along the $x$-axis with velocity $v$. We assume that the surface may be modelled by a system of noninteracting springs. Their elastic, damping and inertial properties are described by the coefficients $k, \gamma$ and $m$. These are defined as follows: $k d x$ and $\gamma d x$ give the elastic and viscous force per unit length along the cylinder axis, $m d x$ gives correspondingly the linear mass density of the springs (i.e. their mass per unit length along this axis). The viscous plane becomes deformed in the range $x_{-} \leq x \leq x_{+}$due to the mass of the cylinder. For deformation rates small as compared with the vertical speed of sound, Hooke's law is valid and one has the equation of motion which describes the behaviour of the surface

$$
m \ddot{y}(x)+\gamma \dot{y}(x)+k y(x)=f(x, t),
$$

where $f$ is the force density ( $f d x$ gives the force per unit length along the cylinder axis) which acts on the plane in the region of contact. Outside of the contact area the force density is zero. In our model we neglect lateral couplings of the springs (for justification of these assumptions see the Appendix). We also assume that no surface waves are excited on the plane, i.e. that the condition of the overdamped motion of the surface

$$
\frac{m}{k}<\frac{1}{4} \frac{\gamma^{2}}{k^{2}}
$$

holds true [11]. Since we are interested in the steady state, i.e. time independent behavior, we do not need to explicitly consider the tangential interactions between the cylinder and the surface. An arbitrarily small tangential force assures that the cylinder does not slide. If one, however, is interested in accelerated motion of the cylinder, tangential forces need to be considered.

By geometrical considerations we find for the shape $y(x)$ of the surface of the deformed plane in the contact area:

$$
y(x)=R-h-\sqrt{R^{2}-\left(x-x_{c}\right)^{2}}, \quad x_{-} \leq x \leq x_{+},
$$

where $x_{c}$ is the $x$-coordinate of the center and $h=$ $-y_{\min }=-y\left(x_{c}\right)$ is the penetration depth of the cylinder. For $\left|x-x_{c}\right| \ll R$ we approximate (3) by

$$
y(x)=\frac{\left(x-x_{c}\right)^{2}}{2 R}-h .
$$

The center of the cylinder moves with constant velocity $v$, i.e. $x_{c}=v t$. Hence, the time derivatives of $y(x)$ read

$$
\begin{aligned}
& \dot{y}(x)=-\dot{x}_{c} \frac{x-x_{c}}{R}=-v \frac{x-x_{c}}{R} \\
& \ddot{y}(x)=\frac{v}{R} \dot{x}_{c}=\frac{v^{2}}{R}=\text { const. }
\end{aligned}
$$

The compression force exerted by the plane to the cylinder is

$$
F_{N}=-\int_{x_{-}}^{x_{+}} f(x) d x
$$

(For simplicity of notation here and in what follows we notate the forces, energy and torque per unit length of the cylinder, e.g. the total force exerted by the plane to the cylinder is $L F_{N}$.)

The springs at $x_{+}$which at time $t$ get in contact with the cylinder need a separate discussion [12]: At time $t-\delta$ $(\delta \rightarrow 0)$ their velocity is zero while infinitesimal time later $\dot{y}(t+\delta)$ is finite according to (5). This singularity in the velocity distribution may be attributed to a force $F_{N}^{\prime}$, acting at point $x_{+}$. This gives a finite contribution to the total force which can be determined by the following consideration:

In the time interval $d t$ the cylinder moves by $v d t$. So it accelerates springs of total mass mvdt. The total momentum received by these springs is $d p=\dot{y}\left(x_{+}\right) m v d t$, hence,

$$
F_{N}^{\prime}=-\frac{d p}{d t}=-\dot{y}\left(x_{+}\right) m v=\frac{x_{+}-x_{c}}{R} m v^{2} .
$$

The total force $F_{N}+F_{N}^{\prime}$ supports thus the weight of the cylinder:

$$
F_{N}+F_{N}^{\prime}=M g
$$

Substituting $y$ and its time derivatives (Eqs. (4,5, 60) in (1) we get an expression for the force density in the contact area

$$
f(x, t)=\frac{k}{2 R}\left(x-x_{c}\right)^{2}-\frac{\gamma v}{R}\left(x-x_{c}\right)+\frac{m v^{2}}{R}-h k
$$

which has to satisfy the contact condition

$$
f(x, t) \leq 0 \quad \text { for } \quad x_{-} \leq x \leq x_{+}
$$

and which determines the boundaries of the contact area $x_{ \pm}$. In comoving coordinates $\xi=x-x_{c}$ Eq. (10) reads

$$
f(\xi)=\frac{k}{2 R} \xi^{2}-\frac{\gamma v}{R} \xi+\frac{m v^{2}}{R}-h k .
$$

The boundary of the contact area at the front side of the cylinder in the direction of motion is

$$
\xi_{+}=\sqrt{2 R h}
$$

according to geometry. The boundary at the back side is determined by $f\left(\xi_{-}\right)=0$, i.e.

$$
\begin{aligned}
0 & =\frac{k}{2 R} \xi_{-}^{2}-\frac{\gamma v}{R} \xi_{-}+\frac{m v^{2}}{R}-h k \\
\xi_{-} & =\frac{\gamma v}{k}-\sqrt{2 h R+\left(\frac{\gamma^{2}}{k^{2}}-2 \frac{m}{k}\right) v^{2}} .
\end{aligned}
$$


For the self-consistency one needs the condition $\xi_{-}<$ $\sqrt{2 R h}$, i.e. $h(v)>m^{2} v^{2} / 2 \gamma^{2} R$ to be fulfilled, which restricts the velocity $v$ from above [13.

Because of the dissipative properties of the plane the motion of the cylinder corresponds to a loss of mechanical energy per time. Another contribution originates from the instantaneous acceleration of plane material which gets in contact with the plane during the time $d t[12$. Therefore, we find for the energy which is transferred per time from the cylinder to the plane

$$
\begin{aligned}
\dot{E} & =-\int_{\xi_{-}}^{\xi_{+}} d \xi f(\xi) \dot{y}(\xi)-m \frac{\dot{y}^{2}\left(\xi_{+}\right)}{2} v \\
& =\frac{v}{R} \int_{\xi_{-}}^{\xi_{+}} d \xi \xi f(\xi)-m v^{3} \frac{2 h}{R} \\
& =-v F_{R} .
\end{aligned}
$$

Equation (16) defines the force $F_{R}$ which acts against rolling of the cylinder

$$
F_{R}=-\frac{1}{R} \int_{\xi_{-}}^{\xi_{+}} d \xi \xi f(\xi)+m v^{2} \frac{2 h}{R} .
$$

To evaluate $F_{R}$ we need the force $f(\xi)$ given by Eq. (12) with the penetration depth $h(v)$ which is to be found. The penetration $h(v)$ results from equilibrating the dynamical resistance of the surface with the weight of the cylinder (9)

$$
M g=-\int_{\xi_{-}}^{\xi_{+}} d \xi\left[\frac{k}{2 R} \xi^{2}-\frac{\gamma v}{R} \xi+\frac{m v^{2}}{R}-h k\right]+\frac{m v^{2}}{R} \sqrt{2 h R}
$$

with the boundaries (13) and (15). Equation (18) is an implicit equation for $h$.

We consider first the limit of small velocities. In this limit Eq. (18) may be solved as a perturbation expansion, $h=h^{(0)}+h^{(1)} v+h^{(2)} v^{2}+\cdots$. It is more convenient, however, to solve (18) with respect to an expansion of $\xi_{+}$:

$$
\xi_{+}=\xi_{+}^{(0)}+v \xi_{+}^{(1)}+v^{2} \xi_{+}^{(2)}+\cdots
$$

Using Eqs.(13), (15) and (19) one can further write the small- $v$ expansion for $\xi_{-}$:

$$
\xi_{-}=-\xi_{+}^{(0)}+v\left(\frac{\gamma}{k}-\xi_{+}^{(1)}\right)-v^{2}\left(\frac{\lambda}{2 \sqrt{2 h R}}+\xi_{+}^{(2)}\right)+\cdots
$$

where

$$
\lambda \equiv\left(\frac{\gamma^{2}}{k^{2}}-2 \frac{m}{k}\right)
$$

Substituting Eqs. (19,20) into Eq. (18) and using (13) one can solve it perturbatively to find the front boundary

$$
\xi_{+}=\left(\frac{3 R}{2 k} M g\right)^{1 / 3}-\frac{\lambda v^{2}}{4 \xi_{+}^{(0)}}+\cdots
$$

where

$$
\xi_{+}^{(0)}=\left(\frac{3 R}{2 k} M g\right)^{1 / 3}
$$

denotes the front boundary for the static case. Then from (20) the rear boundary follows

$$
\xi_{-}=-\xi_{+}^{(0)}+v \frac{\gamma}{k}-\frac{\lambda v^{2}}{4 \xi_{+}^{(0)}}+\cdots
$$

Correspondingly, the penetration depth reads:

$$
h(v)=h_{0}-\frac{\lambda v^{2}}{4 R}+\cdots
$$

where

$$
h_{0} \equiv h^{(0)}=\left(\xi_{+}^{(0)}\right)^{2} / 2 R
$$

is the static penetration depth. From Eqs. (24) and (21) it follows that the penetration depth $h$ decreases with increasing velocity.

Using the obtained expansions for $\xi_{+}$and $\xi_{-}$it is straightforward to calculate the rolling friction force. Substituting (10), 21) and (23) into Eq. (17) one finally arrives at an expression for the rolling friction torque, $\mathcal{M}=R F_{R}$

$$
\begin{aligned}
\mathcal{M} & =\mu_{\text {roll }} M g \\
\mu_{\text {roll }} & =\frac{\gamma}{k} v-\frac{3 \lambda}{4 \xi_{+}^{(0)}} v^{2}+\cdots
\end{aligned}
$$

As it follows from Eq. (27), in the limit of small velocities the leading linear term depends only on the viscous and elastic constants and does not depend on the inertial properties of the material, characterized by $m$. This means that in this regime the inertial effects in the deformation process of the plane are negligible. The second nonlinear term takes into account via $m / k$ in $\lambda$ the inertial effects up to $\mathcal{O}\left(v^{2}\right)$. It is also interesting to note, that while the linear term does not depend on the radius of the body, the nonlinear term depends (via $\xi_{+}^{(0)}$ ) on both, the radius of the cylinder and its mass per unit length $M$.

In the general case Eq. (18) has to be solved numerically. The velocity dependence of the penetration depth $h$ and of the rolling friction coefficient $\mu_{\text {roll }}$ are shown in Fig. 2 and Fig. 3. Calculations were performed for a steel cylinder of radius $R=0.1 \mathrm{~m}$, and of mass per unit length 
$M=250 \mathrm{~kg} \mathrm{~m}^{-1}$ rolling on the rubber surface with the following material parameters: $m=100 \mathrm{~kg} \cdot \mathrm{m}^{-2}$, $k=10^{7} \mathrm{~kg} \cdot \mathrm{m}^{-2} \cdot \mathrm{s}^{-2}, \gamma=5 \cdot 10^{5} \mathrm{~kg} \cdot \mathrm{m}^{-2} \cdot \mathrm{s}^{-1}$. These values were obtained from density and elastic constants of rubber 114]; the viscous constant was estimated from the restitution coefficient for colliding rubber spheres, similarly as in [9], where the details of this estimate are given.

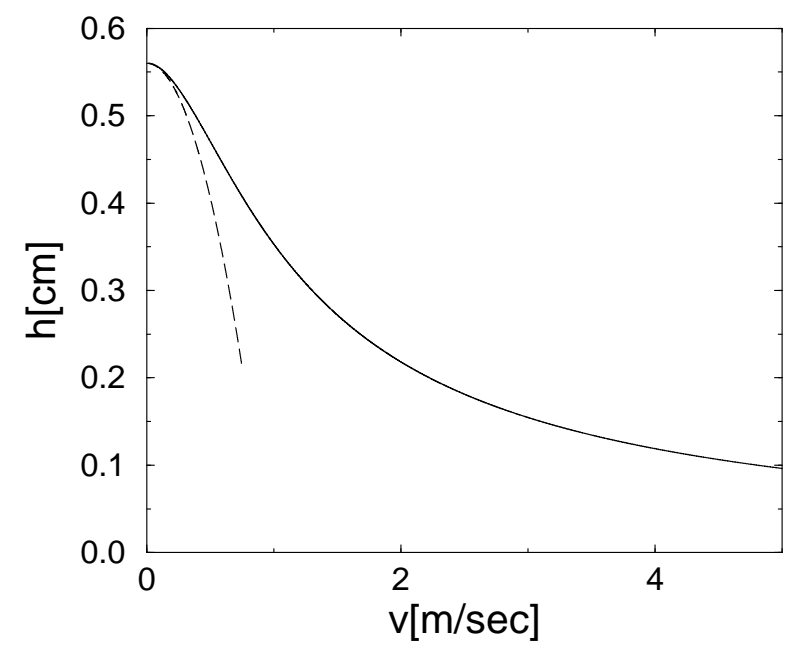

FIG. 2. The penetration depth $h$ as a function of the velocity $v$ according to the numerical solution of Eq. (18). The dashed line shows the approximation (24).

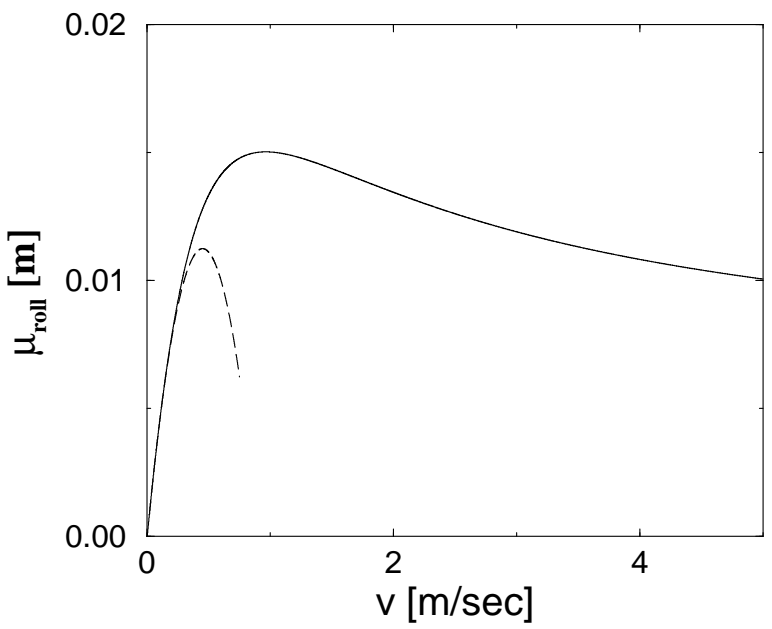

FIG. 3. The rolling friction coefficient $\mu_{\text {roll }}$ over the velocity $v$ (numerical solution). The dashed line shows the approximation (27).

As shown in the figures, at high velocities the depth $h$ decreases and the rolling friction coefficient reaches a maximal value of

$$
\mu_{\text {roll }}^{*}=\frac{1}{3} \frac{\gamma^{2}}{k^{2}} \frac{\xi_{+}^{(0)}}{\lambda}
$$

at

$$
v^{*}=\frac{2}{3} \frac{\gamma}{k} \frac{\xi_{+}^{(0)}}{\lambda}
$$

For velocities $v>v^{*}$ the rolling friction coefficient decreases with increasing velocity. For particular parameters used here one obtains $\mu_{\text {roll }}^{*}=1.125 \cdot 10^{-2} \mathrm{~m}$ and $v^{*}=0.4500 \mathrm{~ms}^{-1}$, which are in a reasonable agreement with the numerically obtained values (see Figs. 23 and 3).

Note that with increasing velocity the rear boundary $\xi_{-}$shifts in positive direction and approaches the front boundary $\xi_{+}$. The contact area between the surface and the cylinder thus decreases and at some critical velocity it shrinks to a point (a line along the cylinder axis). For velocities larger than this critical one the weight of the cylinder is sustained by the force $F_{N}^{\prime}$, acting at a single point at the front boundary $\xi_{+}$.

\section{SUMMARY AND DISCUSSION}

We investigated the rolling motion of a hard cylinder on a viscous plane. The elastic, viscous and inertial properties of the plane were modelled by a system of uncoupled springs which are characterized by linear elastic and viscous coefficients and mass density. For small velocity of the rolling cylinder we determined the velocity expansion of the rolling friction coefficient analytically up to second order. For larger velocities the rolling friction was determined numerically.

In the range of low velocity our analysis shows increasing rolling friction coefficient with increasing velocity. At a certain velocity the coefficient reaches its maximal value and decreases when the velocity is further increased.

In the low velocity regime where the rolling friction coefficient rises linearly with the velocity of the cylinder its value depends on the ratio of the viscous and elastic constants. For high velocities one notes nonlinear dependence on the cylinder's mass, radius and on the material constants of the surface.

We also analyzed the dependence of the penetration depth (i.e. the depth at which the cylinder sinks into the bulk of the surface) on the cylinder velocity. We found that the penetration depth decreases with increasing velocity, i.e. that the rolling cylinder emerges upwards when its velocity increases.

\section{ACKNOWLEDGMENTS}

Frank Spahn is acknowledged for discussion. This work was supported by Deutsche Forschungsgemeinschaft (Po 472/3-2 and 472/5-1).

\section{APPENDIX: LATERAL COUPLINGS}

In our model we assumed that the viscous surface is composed of springs which are not coupled in lateral (horizontal) direction. This simplification may limit the validity of the model since we ignore lateral interactions 
in the plane, which obviously exist in real systems and which cause the tangential elasticity of the surface. In the appendix we want to study in more detail the range of validity of our model.

Lateral interactions of the springs may be taken into account if instead of Eq. (11) one uses

$$
m \ddot{y}(x)+\gamma \dot{y}(x)+k y(x)-d \frac{\partial^{2}}{\partial x^{2}} y=f(x, t),
$$

where the constant $d$ describes the lateral coupling between the springs. With the dimensionless variables $\hat{y}=y / R, \hat{x}=x / R$ and $\hat{t}=t /(R / v)$ the left-hand side of Eq. (A1) reads

$$
\frac{m v^{2}}{R} \frac{\partial^{2}}{\partial \hat{t}^{2}} \hat{y}(\hat{x})+\gamma v \frac{\partial}{\partial \hat{t}} \hat{y}(\hat{x})+k R \hat{y}(\hat{x})-\frac{d}{R} \frac{\partial^{2}}{\partial \hat{x}^{2}} \hat{y}(\hat{x}) .
$$

Thus we conclude that if the radius of the cylinder satisfies the condition

$$
R^{2} \gg \frac{d}{k}
$$

the term describing the lateral coupling is negligible and our model is valid.

The value of $\sqrt{d / k}$ is a characteristic length of influence of lateral couplings. If the above condition (A3) is satisfied, one can apply Eq. (11) for almost the entire contact region, except for the (small) region around $\xi_{+}$. In these region the deformation of the surface may differ from the idealized shape which, as we assumed, results from pure geometrical considerations. Instead the range of deformation of the surface is slightly enhanced by a characteristic size $\sim r_{0}$ due to lateral couplings between the springs. If the cylinder is at rest or if it moves very slowly, similar discussion applies to the small region $\sim r_{0}$ around the rear point $\xi_{-}$. Consider first the motion of the surface in a region around $\xi_{+}$.

Since $\xi_{+} \gg r_{0}$ the region of the characteristic size $\sim r_{0}$ may be considered as a point, so that the total force acting on this region may be attributed to the single point $\xi_{+}$. The dissipation in this region (which is finite) is, therefore, attributed to the point $\xi_{+}$too. Coarsegraining, therefore, results in the force $F_{N}^{\prime}\left(\xi_{+}\right)$acting at the point $\xi_{+}$.

We will estimate the characteristic size $r_{0}$ : If we take into account lateral couplings in the region $r_{0}$ around $\xi_{+}$ there is no contact between the surface and the cylinder (free surface condition). The derivative $y^{\prime} \equiv \partial y / \partial x$ changes from zero (condition of the undisturbed plane) to $y^{\prime} \approx y^{\prime}\left(\xi_{+}\right) \approx \xi_{+} / R$ at the point of contact, which follows from the geometry of the system (see Fig. 1). Thus, in this region one estimates $y^{\prime \prime} \equiv \partial^{2} y / \partial x^{2} \sim \xi_{+} / R r_{0}$. Similarly, the characteristic value of $y$ in this region reads $y \sim r_{0} \cdot y^{\prime} \sim r_{0} \cdot \xi_{+} / R$ and, correspondingly, the characteristic values $\dot{y}=\dot{y}\left(\xi_{+}\right)=-v \xi_{+} / R$ and $\ddot{y}=v^{2} / R$ follow from Eqs. (5), (5). Then we write the condition of the free surface in this transient region,

$$
m v^{2} / R-\gamma v \xi_{+} / R+k r_{0} \xi_{+} / R-d \cdot \xi_{+} / R r_{0}=0
$$

to estimate the size of the region:

$$
r_{0}=\sqrt{\frac{d}{k}+\left(\frac{m v^{2}}{2 k \xi_{+}}-\frac{\gamma v}{2 k}\right)^{2}}-\left(\frac{m v^{2}}{2 k \xi_{+}}-\frac{\gamma v}{2 k}\right)
$$

which yields $r_{0}=\sqrt{d / k}$ for the static case. Hence, the condition for coarse-graining, $r_{0} \ll \xi_{+}$, reads

$$
\frac{d}{k} \ll \xi_{+}^{2}+\frac{m}{k} v^{2}-\frac{\gamma}{k} v \xi_{+} .
$$

We want to discuss the consequences of the assumption (A6) or of the assumption $\xi_{+}^{2} \gg d / k$ (the later condition follows from the former one, unless the velocity is too high, i.e. unless $v \gg \xi_{+} \sqrt{k / m}$ ). In this case the force $F_{N}^{\prime}\left(\xi_{+}\right)$which acts at the point $\xi_{+}$reads

$$
F_{N}^{\prime}\left(\xi_{+}\right)=\int_{\xi_{+}-\delta}^{\xi_{+}+\delta} f(\xi, t) d \xi
$$

where $\delta$ is of the order (say somewhat larger) than $r_{0}$, and we can write for the different terms in the left-hand side of Eq. (A1):

$$
\begin{aligned}
& \int_{\xi_{+}-\delta}^{\xi_{+}+\delta} m \ddot{y} d x=m \int_{\xi_{+}-\delta}^{\xi_{+}+\delta} \frac{d \dot{y}}{d t} d x=m \int_{\xi_{+}-\delta}^{\xi_{+}+\delta} d \dot{y} \frac{d x}{d t} \\
& =m v \int_{\xi_{+}-\delta}^{\xi_{+}+\delta} d \dot{y}=m v\left[\dot{y}\left(\xi_{+}+\delta\right)-\dot{y}\left(\xi_{+}-\delta\right)\right] \\
& =-m v \dot{y}\left(\xi_{+}\right)=m v^{2} \xi_{+} / R
\end{aligned}
$$

where we take into account that $\dot{y}\left(\xi_{+}+\delta\right)=0$ (the surface is at rest) and that $\dot{y}\left(\xi_{+}-\delta\right)=\dot{y}\left(\xi_{+}\right)$on the coarsegrained scale. Similarly, using the above estimate of $y(x)$ in the transient region, we obtain the coarse-grained estimates:

$$
\gamma \int_{\xi_{+}-\delta}^{\xi_{+}+\delta} \dot{y} d x=\gamma v\left[y\left(\xi_{+}+\delta\right)-y\left(\xi_{+}-\delta\right)\right] \sim-\gamma v r_{0} \xi_{+} / R
$$

and

$$
k \int_{\xi_{+}-\delta}^{\xi_{+}+\delta} y d x \approx k \int_{\xi_{+}-\delta}^{\xi_{+}+\delta} y^{\prime}\left(\xi_{+}\right) x d x \sim 2 k r_{0} \xi_{+}^{2} / R .
$$

Finally, the last term reads

$$
\begin{aligned}
& -d \cdot \int_{\xi_{+}-\delta}^{\xi_{+}+\delta} y^{\prime \prime} d x=-d \cdot\left[y^{\prime}\left(\xi_{+}+\delta\right)-y^{\prime}\left(\xi_{+}-\delta\right)\right] \\
& =d \cdot y^{\prime}\left(\xi_{+}\right)=d \cdot \xi_{+} / R .
\end{aligned}
$$

As it follows from Eqs. (A8 A11) the second and third terms, proportional to $r_{0}$, vanish on the coarse-grained scale. The fourth term does contribute to $F_{N}^{\prime}\left(\xi_{+}\right)$on the 
coarse-grained level, but it does not depend on the velocity $v$. It may be taken into account within the general scheme of calculation of the rolling friction given above. Namely, with this term included, one obtains, e.g. for the front boundary

$$
\xi_{+}^{(0)}=\left\{\frac{3 R M g}{2 k}\left[1+\frac{3}{2} \frac{(d / k)}{\left(\xi_{+}^{(0)}\right)^{2}}\right]\right\}^{1 / 3} \approx\left(\frac{3 R M g}{2 k}\right)^{1 / 3}
$$

where the condition $\xi_{+}^{2} \gg d / k$ was used. Similarly, the impact of this term on the other expressions obtained previously is negligible, i.e. it is of the order $(d / k) /\left(\xi_{+}^{(0)}\right)^{2} \ll 1$ under the coarse-grained condition. Thus, we conclude that the fourth term in $F_{N}^{\prime}\left(\xi_{+}\right)$, which accounts for the lateral interactions may be also neglected. This gives the result

$$
F_{N}^{\prime}\left(\xi_{+}\right)=m v^{2} \xi_{+} / R
$$

Similar considerations may be performed for the dissipation in the transient region. Skipping the details of the analysis (very similar to that for $F_{N}^{\prime}\left(\xi_{+}\right)$), we give the final result:

$$
\int_{\xi_{+}-\delta}^{\xi_{+}+\delta} f(\xi, t) \dot{y}(\xi) d \xi=-m \frac{\dot{y}^{2}\left(\xi_{+}\right)}{2} v
$$

which describes the energy loss in the point $\xi_{+}$on the coarse-grained scale.

Using the same reasoning one can consider the region $\sim r_{0}$ around the rear point $\xi_{-}$to conclude that under the condition $\xi_{+}^{2} \gg d / k$ its contribution to the total force and to the dissipation is negligible: Indeed, the region $\sim r_{0}$ in the rear part of the contact area may influence the motion of the cylinder only for very small velocities $v$ when $\left|\xi_{+}\right|-\left|\xi_{-}\right| \sim r_{0}$. However, contribution to the force and dissipation from this rear region is proportional to $r_{0}$ and therefore may be neglected.

Thus, we conclude that our simplified model of the viscous surface as a system of linear uncoupled springs may be adequate for real systems and it may be used to model the rolling friction phenomenon.

[1] H. Czichos, Tribology Elsevier (Amsterdam, 1978); R. H. Bentall and K. L. Johnson, Int. J. Mech. Sci. 6, 389 (1967); F. T. Barwell, Bearing Systems: Principles and Practice, Oxford Univ. Press (Oxford, 1979), p. 312; A. Z. Szeri, Tribology-Friction, Lubrication, and Wear, Hemisphere (Washington, 1980), p. 401; D. Dowson, C. M. Taylor, T. H. C. Childs, M. Godet, and G. Dalmaz (Eds.),
Dissipative Processes in Tribology, Elsevier (Amsterdam, 1994); H. Krause and H. Lehna, Wear 119, 153 (1987); G. P. Shpenkov, Friction Surface Phenomena, Elsevier (Amsterdam, 1995); E. Rabinowicz, Friction and Waer of Materials, Wiley (New York, 1965); K.-H. zum Gahr, Microstructure and Wear of Material, Elsevier (Amsterdam, 1987); F. W. Carter, Proc. Roy. Soc. A 112, 151 (1926); A. Domenéch, T. Domenéch, and J. Cebrián, Am. J. Phys. 55, 231 (1986); K. R. Eldredge and D. Tabor, Proc. Roy. Soc. A 229, 180 (1955); D. G. Evseev, B. M. Medvedev, G. G. Grigoryan, and O. A. Ermolin, Wear 167, 33 (1993); Y. F. Li and A. Seireg, J. TribilogyTransactions of the ASME 111, 386 (1989); S. Lingard, Wear 117, 109 (1987); D. E. Shaw and F. J. Wunderlich, Am. J. Phys. 52, 997 (1984); P. H. Vo, Proceedings of the SPIE - Int. Soc. Opt. Engin. 1998, 141 (1993); K. Weltner, Am. J. Phys. 55, 937 (1987); J. Witters and D. Duymelink, Am. J. Phys. 54, 80 (1986).

[2] O. Reynolds, Phil. Trans. Roy. Soc. 166, 1 (1874); R. Q. Huang and S. T. Wang, In: Chr. Bonnard (Ed.), Proc. Intern Symp. on Landslides, Lausanne, Juli 10-15, 1988 (1988), p. 187; H. J. Herrmann, G. Mantica, and D. Bessis, Phys. Rev. Lett. 65, 3223 (1990).

[3] S. Vince. Phil. Trans. Roy. Soc. London 75, 165 (1785).

[4] M. Barquins, D. Maugis, J. Blouet, and R. Courtel, Wear 51, 375 (1978); B. V. Deryaguin and Y. P. Toporov, Progr. in Surface Sci. 45, 317 (1994); K. Kendall, Wear 33, 351 (1975); K. N. G. Fuller and A. D. Roberts, J. Phys. D 14, 221 (1981); A. D. Roberts and A. G. Thomas, Wear 33, 45 (1975).

[5] B. V. Deryaguin and V. P. Smilga, Progr. in Surface Sci. 45, 108 and 296 (1994); H. Prashad, J. TribilogyTransactions of the ASME 110, 448 (1988); P. V. Nazarenko, V. F. Labunets, V. S. Pilyavsky, and M. N. Popovich, Dopovidi Akademii Nauk Ukrainskoi RSR A 2, 68 (1986).

[6] R. L. Chaplin and P. B. Chilson, Wear 107, 213 (1986); Q-g. Song; Am. J. Phys. 56, 1145 (1988); R. L. Chaplin and M. G. Miller, Am. J. Phys. 52, 1108 (1984).

[7] J. A. Greenwood, H. Minshall, and D. Tabor, Proc. Roy. Soc. A 259, 480 (1961).

[8] D. Tabor, Proc. Roy. Soc. A 229, 198 (1955); 43, 1055 (1952); D. Atack and D. Tabor, Proc. Roy. Soc. A 246, 539 (1958); R. C. Drutowski, In R. Davies (Ed.), Proc. Symp. Friction and Wear, Elsevier (Amsterdam, 1959), p. 17.

[9] N. V. Brilliantov and T. Pöschel, Europhys. Lett 42, 511 (1998).

[10] N. V. Brilliantov, F. Spahn, J.-M. Hertzsch, and T. Pöschel, Phys. Rev. E 53, 5382 (1996).

[11] For the case of lower damping the situation appears to be more complicated: Surface waves of the plane are excited and it may happen that the area of contact is not continuous, i.e. the sphere and the surface touch in multiple regions $x_{-}^{(1)} \leq x \leq x_{+}^{(1)}$ and $x_{-}^{(2)} \leq x \leq x_{+}^{(2)}<x_{-}^{(1)}$, etc. This complicated behaviour which resembles Schallamach waves is excluded if we require overdamped motion of the plane. Actually, it may be shown that more weak than Eq. (8) condition, $m / k<\gamma^{2} / 2 k^{2}$, should be satisfied to exclude the multiple contact areas. This cor- 
responds to surface waves appearing in the rear part of the plane which has been already passed by the rolling cylinder. Such kind of waves will not affect our calculations.

[12] In real systems where the surface also has a tangential elasticity, which may be modelled by the lateral coupling between the springs, one should consider a transient region of the plane of some finite extent. For the case of large radius of the cylinder, compared to the penetration depth, and for large vertical elasticity, compared to the tangential elasticity, the transient region is small. One can then attribute the force and dissipation occuring in this region to a single point. Since the region is small, the viscous losses are negligible compared to the energy required to accelerate this part of the surface material. More details are given in the Appendix.

[13] If this condition does not hold the cylinder is sustained only by the singular force $F_{N}^{\prime}$. This situation reminds to water skiing, when all the weight of the skier is sustained by the inertia of the water. In this case the lateral coupling of springs ignored in the present model is important.

[14] Landolt-Börnstein. New Series, vol. V/1b. Springer Verlag, (Berlin, 1982). 